\title{
Video-assisted thoracic surgery lung resection after endobronchial valve placement
}

\author{
Juan C. Garzon, MD, Calvin S. H. Ng, MD, Tak Wai Lee, MD, and Anthony P. C. Yim, MD, Hong Kong, China
}

(2) ndobronchial valve (EBV) placement has emerged as an alternative approach for treating patients with severe emphysema. ${ }^{1}$ The improvements in lung function after EBV placement, which allows video-assisted thoracic surgery (VATS) lung resection in a previously nonsurgical candidate, have not been reported. We present a case of VATS lung resection after EBV placement in a patient with severe emphysema to illustrate clinical and surgical aspects in this new category of patients.

\section{Clinical Summary}

A 70-year-old female ex-smoker, who was known to have severe emphysema with multiple admissions for exacerbation, was treated with EBVs. The EBVs were placed in both lower lobes including the apical segments, thereby achieving total bilateral lower lobe collapse (Figure 1). During the following year, she had significant clinical improvement confirmed by serial pulmonary function tests showing an increase in forced expiratory volume in 1 second from $0.61 \mathrm{~L}$ ( $39 \%$ predicted) to $1.46 \mathrm{~L}$ ( $75 \%$ predicted), and in forced vital capacity from $1.67 \mathrm{~L}$ to $2.08 \mathrm{~L}$. The diffusing capacity of lung for carbon monoxide of $7.3 \mathrm{~mL} \cdot \mathrm{mm} \mathrm{Hg} \cdot \min (50 \%$ predicted) remained the same. However, at 1 year's follow-up after the EBV procedure, an incidental finding of a new solitary pulmonary nodule in the left upper zone was detected on the chest radiograph. Computed tomography (CT) showed a $1.5-\mathrm{cm}$ solitary pulmonary nodule arising from the left upper lobe with no mediastinal lymphadenopathy. The collapsed lower lobes with 4 in situ EBVs were also visualized on CT (Figure 1). Because of the clinical suspicion of primary lung malignancy in an ex-smoker, surgical exploration with frozen section of the lung nodule was planned. Fine-needle aspiration cytology of the lung nodule was considered but not pursued, not only because of the risk of a pneumothorax but also because of the unreliability of a negative result in a small nodule. ${ }^{2}$ During VATS exploration of the left lung, both lower lobes were fully collapsed and a pulmonary nodule was found to be located deep in the posterior segment of the left upper lobe. A wide wedge

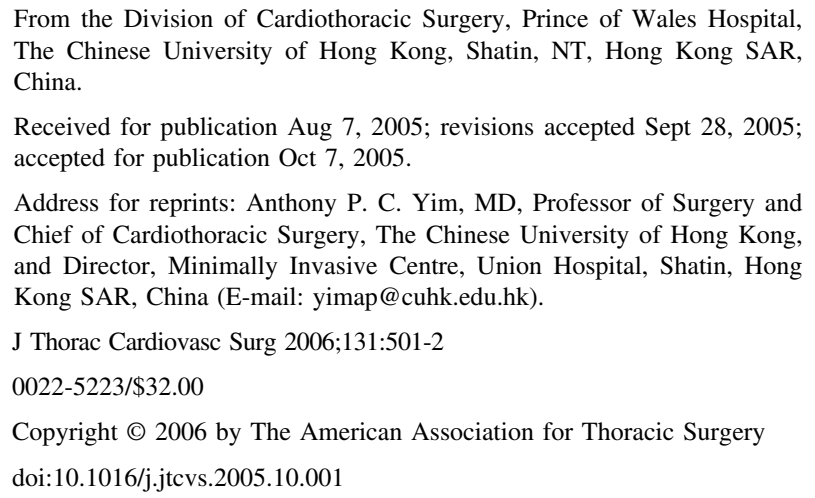

resection of the nodule was performed, and pathologic examination confirmed a granulomatous nodule without evidence of malignancy. Results of subsequent tuberculosis culture was also negative. The patient made an uneventful recovery from surgery.

\section{Discussion}

In general, patients with severe emphysema have a higher risk for lung cancer. The prognosis, in terms of survival at 5 years, for patients with severe emphysema is similar for patients with lung cancer. ${ }^{3}$ Given the poor survival of patients with severe emphysema with or without lung cancer, the real significance of a new pulmonary nodule in this group of patients is often debatable. Some patients with emphysema simply would not tolerate lung surgery, whereas the wisdom of performing high-risk lung resection in others with limited life expectancy requires justification and further investigation. At present, it is difficult to predict whether the lung cancer or the underlying emphysema will be the terminal condition. Over the past few years, EBVs have been offered to patients with advanced emphysema, and the appearance of suspicious nodules in these patients who have undergone EBV placement has important implications.

If the nodule is located in the collapsed parenchyma, it might be difficult to identify when the collapsed lung has density and enhancement similar to soft tissue tumors. Usually, lung nodule enhancement of more than 15 Hounsfield units on high-resolution chest CT is strongly suggestive of malignancy ${ }^{4}$; however, differentiation between atelectasis and malignancy could be difficult and surgical exploration may be necessary to rule out malignancy. Furthermore, a recent study by Matsuoka and colleagues ${ }^{5}$ showed that CT findings cannot be used reliably to discriminate between malignant and benign pulmonary nodules in patients with associated severe emphysema. In addition, if the nodule appears in the noncollapsed parenchyma, such as in our patient, there will be changes in the pulmonary anatomy after EBV placement, which can make the proper localization and adequate intraoperative palpation of the lesion more difficult.

Placement of EBVs before lung resection for pulmonary nodule may significantly improve pulmonary function in patients with severe emphysema, thereby reducing the risk of surgery, or even allow previously nonsurgical candidates to undergo VATS lung resection. Our patient had post-EBV forced expiratory volume in 1 second of 1.46 ( $75 \%$ predicted), which would have allowed for lobectomy if the nodule were malignant.

\section{References}

1. Yim AP, Hwong TM, Lee TW, et al. Early results of endoscopic lung volume reduction for emphysema. J Thorac Cardiovasc Surg. 2004; 127:1564-73. 

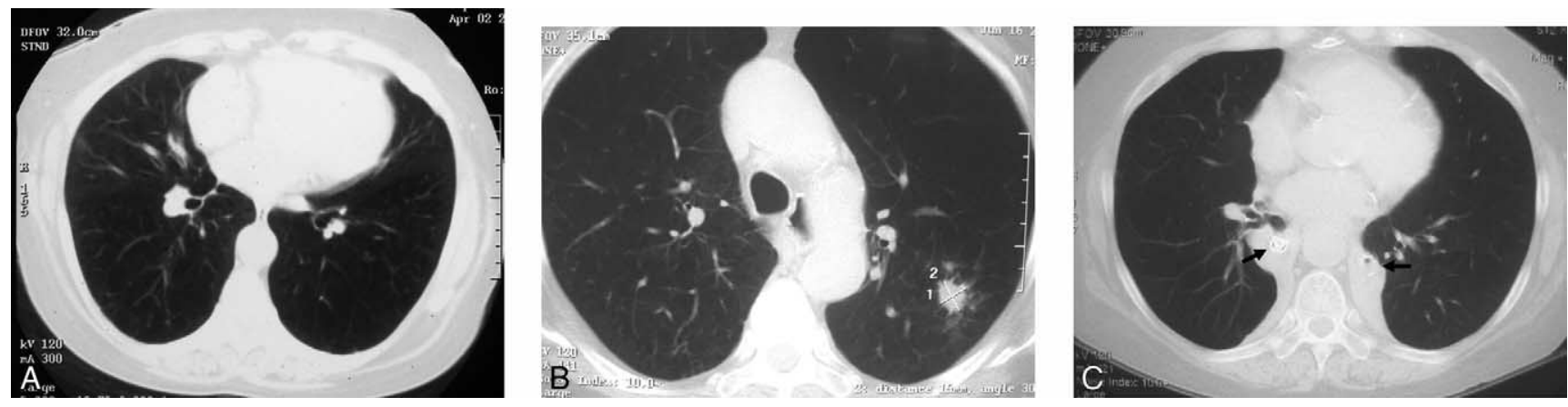

Figure 1. A, High-resolution chest computed tomography (CT) of the lower lobes before endobronchial valve (EBV) placement. B, High-resolution chest CT showing the nodule located in the posterior segment of the left upper lobe. C, Bilateral lower lobe collapse after EBV placement, and EBVs in the lower lobe bronchus, bilaterally (arrows).

2. Ost D, Fein AM, Feinsilver SH. The solitary pulmonary nodule. $N$ Engl J Med. 2003;348:2535-42.

3. Littman AJ, Thornquist MD, White E, et al. Prior lung disease and risk of lung cancer in a large prospective study. Cancer Causes Control. 2004;15:819-27.
4. Swensen SJ, Viggiano RW, Midthun DE, et al. Lung nodule enhancement at CT: multicenter study. Radiology. 2000;214:73-80.

5. Matsuoka S, Kurihara Y, Yagihashi K, Niimi H, Nakajima Y. Peripheral solitary pulmonary nodule: CT findings in patients with pulmonary emphysema. Radiology. 2005;235:266-73.

\section{Internal thoracic vein aneurysm presenting as an anterior mediastinal mass}

G. Hossein Almassi, MD, Milwaukee, Wis

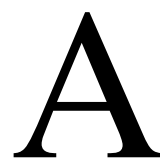

nterior mediastinal masses most commonly are associated with thymic, thyroid, parathyroid, and lymphatic origin. Excluding aortic disease, vascular origin for anterior mediastinal masses is not common. We present a case of an aneurysm of the internal thoracic vein presenting as an enlarging anterior mediastinal mass.

\section{Clinical Summary}

A 52-year-old woman underwent a triple arthrodesis of the right foot for orthopedic injuries sustained in a car crash 16 months

\footnotetext{
From the Division of Cardiothoracic Surgery, Medical College of Wisconsin, Milwaukee Wis.

Received for publication July 7, 2005; revisions received Sept 21, 2005; accepted for publication Sept 28, 2005.

Address for reprints: G. Hossein Almassi, MD, Professor, Division of Cardiothoracic Surgery, Medical College of Wisconsin, 9200 W. Wisconsin Ave, Milwaukee, WI 53226 (E-mail: halmassi@mcw.edu).

J Thorac Cardiovasc Surg 2006;131:502-3

$0022-5223 / \$ 32.00$

Copyright (C) 2006 by The American Association for Thoracic Surgery doi:10.1016/j.jtcvs.2005.09.043
}

earlier. One week later while she was at home, acute shortness of breath with wheezing developed. Results of a contrast computed

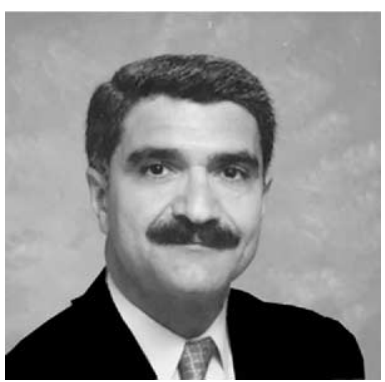

Dr H. Almassi tomographic (CT) scan of the chest were negative for pulmonary embolism. CT scan, however, revealed a $2.2-\mathrm{cm}$ round mass anterior to the superior vena cava and adjacent to the ascending aorta. There was no mediastinal lymphadenopathy (Figure 1, A). The patient was referred for further treatment. Review of the CT scans obtained at the time of the car crash demonstrated the presences of this mass, albeit, smaller (Figure 1, $B$ ).

The patient's chest was explored through a limited partial upper sternotomy incision. The mass was found to be an aneurysm of the right internal thoracic vein just before its connection to the innominate vein (Figure 2). The right internal thoracic vein was ligated on both sides of the aneurysm, and the mass was excised. The patient made an uneventful recovery. Pathologic examination was negative for malignancy.

\section{Discussion}

Anterior mediastinal masses may present a challenge in diagnosis. Depending on the age of the patient and symptoms, these may 